\title{
BAMBINI INFELICI E FAMIGLIE INFRANTE NEL CINEMA DI REGIME
}

\section{Meris Nicoletto}

Riassunto: Il saggio focalizza la sua attenzione sul tema dell'adulterio femminile, considerato argomento tabù dalla censura e dalla propaganda fascista, e sulle ripercussioni che esso provoca sui figli. Piccola mia di Eugenio De Liguoro e Il canale degli angeli di FrancescoPasinetti, pellicole uscite a metà anni Trenta, bypassano la tematica incandescente del tradimento extraconiugale con il ravvedimento finale delle protagoniste che tornano al loro ruolo di matres familiae. In particolare il film di Pasinetti anticipa di quasi dieci anni la storia raccontata da I bambini ci guardano di Vittorio De Sica: Bruno, il bimbo protagonista, vive con partecipazione sofferta la nascita di un affetto tra la propria madre e un marinaio. La possibile perdita della figura materna viene somatizzata dal bambino in gesti e azioni fatte di silenzi, di avvicinamenti al padre, di furtivi sguardi verso i due amanti. Il tutto poi si scioglie nel finale quando il marinaio decide di partire e la madre di rimanere con il figlio e il marito. Ne I bambini ci guardano, Pricò non è più in grado di salvare il matrimonio dei genitori perché la famiglia non è più una cellula salda e integra, come il regista dimostra superando ipocrisie ideologiche e radiografando pulsioni sociali ed esistenziali nuove. Lo sguardo del bimbo è lo stesso di Bruno, ma con una messa a fuoco più nitida di quanto accade attorno a lui. A nulla valgono $\mathrm{i}$ suoi tentativi di nascondere al padre $\mathrm{i}$ continui tradimenti della madre, che alla fine rinuncia al figlio in nome della felicità personale. E nulla può il bimbo per richiamare il padre ai suoi doveri di pater familias. In seguito al trauma dell'abbandono della madre e del suicidio del padre Pricò diventa adulto e sceglie di rimanere in collegio, pur avendo di fronte a sé una madre disponibile a prendersi ancora cura di lui. Il suo dolore è troppo forte per perdonare la donna che lo ha tradito nei suoi affetti più profondi.

\section{L'adulterio femminile come causa di disgregazione della famiglia}

Per tutto il corso degli anni Trenta il regime propagandò "un modello tradizionale e cattolico di coppia e di famiglia, finalizzato alla procreazione e sottoposto all'autorità maschile e paterna" (Dogliani 106). La madre ideale era colei che era disposta a sacrificare le proprie aspirazioni "per il bene spirituale del marito e dei figli" (Dau Novelli 39). Alla moglie 'vestale della casa' faceva da pendant il marito, pater familias esemplare, virile e devoto, con la stessa abnegazione della madre, alla cura della prole. Con l'accen- 
tuarsi della crisi demografica, verso la fine degli anni Venti, il regime, ormai consapevole della funzione coagulante dei valori tradizionali per costruire uno Stato totalitario, portò avanti la sua battaglia a favore della natalità puntando proprio sul "mito della donna angelicata e dell'uomo virile" (Dau Novelli 42). Questa immagine edulcorata della famiglia, salvo rare eccezioni, veniva promossa dall'industria culturale del regime, in particolare dai documentari e dai cinegiornali dell'Istituto Luce. Il cinema italiano, considerato l' "arma più forte", aveva indubbiamente finalità didattiche, ma era per lo più influenzato dalla produzione americana e budapestina. Per incontrare prodotti filmici meno in sintonia con la politica dell'Italia fascista dobbiamo attendere la fine degli anni Trenta e i primi anni Quaranta in cui avvenne un incremento, seppur contenuto, sia di commedie che di melodrammi ${ }^{1}$, in concomitanza al vuoto lasciato dal cinema americano in seguito alla battaglia per l'autarchia. Eitel Monaco, nominato Direttore della DGC nel 194122, lasciò campo aperto ai produttori e agli autori, che realizzarono sia opere di qualità media, come la commedia d'evasione, genere principe lungo tutti gli anni Trenta, sia opere di nuova concezione, soprattutto nel genere meno praticato del mélo ${ }^{3}$. Il nuovo corso della politica culturale in ambito cinematografico si riscontrò anche nell'apertura da parte della DGC a progetti di produttori non allineati con il regime o comunque disposti a confezionare film che affrontassero tematiche considerate fino a quel momento tabù, quali l'adulterio, il delitto passionale, il suicidio, la maternità illegittima ${ }^{4}$.

${ }^{1}$ Il genere dominante rimane la commedia, un genere che strutturalmente vede i conflitti appianarsi e offrire al pubblico il rassicurante lieto fine. Il melodramma invece è in dissonanza con l'ordine sociale. Giovanna Grignaffini ha sottolineato che la principale causa di "sottrazione di sostanza mitica alla figura materna nel cinema italiano degli anni Trenta" è "strettamente correlata allo scarso peso che al suo interno assume il genere fondato per eccellenza sulla scena materna, cioè il melodramma” (Grignaffini 246). Per una mappatura del genere, attraverso l'analisi di film significativi, si veda il saggio di Lucia Cardone.

2 Luigi Freddi lasciò la Direzione Generale per la Cinematografia nel marzo 1939 per contrasti con il ministro Alfieri. Gli subentrò Vezio Orazi fino all'aprile 1941.

3 Per una trattazione più approfondita sulla produzione melodrammatica del periodo, in particolare sulla figura della donna peccatrice, si rimanda al capitolo $\mathrm{V}$ della mia tesi di dottorato (233-304).

4 Jean A. Gili, nel suo libro Stato fascista e cinematografia, riporta questa testimonianza di Monaco: "Per Ossessione, la battaglia non è stata condotta allo stadio della censura, ma ad uno stadio precedente. Io, Direttore Generale per la 
Si tratta per lo più di melodrammi in cui è evidente la crisi dell'istituzione familiare scaturita da nuove pulsioni femminili che mettono in discussione l'antica concezione di mater familias. L'emancipazione cui la donna era andata incontro, nonostante il regime si prodigasse contraddittoriamente a dipingerla come custode del focolare, si riverberava anche nell'ambito dei rapporti coniugali. Il fascismo considerava il matrimonio indissolubile, ma si mostrava ambiguo nei riguardi della reciproca fedeltà dei coniugi. La moglie era destinata per natura alla procreazione e per questo le si negava, anche nell'ambito del rapporto di coppia, qualsiasi forma di passionalità, perché in netto contrasto con la sua funzione di 'fattrice' di italiani. Le relazioni extraconiugali maschili erano tollerate, purché non mettessero in discussione l'unione familiare'.

Questa premessa è indispensabile per comprendere, oltre che il contesto socio-politico-familiare del periodo, in linea, quasi sempre, con i dettami della chiesa cattolica ${ }^{6}$, la produzione cinematografica che precede l'uscita di un film di rottura della visione stereotipata della famiglia, qual è $I$ bambini ci guardano (1943) di Vittorio De Sica.

\section{L'amore filiale vince l'eros extraconiugale: Piccola mia e Il canale degli angeli}

Se la famiglia è la grande assente sul grande schermo 7 , lo sono di conse-

Cinematografia, ho preso la responsabilità di far concedere a Visconti il finanziamento della Banca Nazionale del Lavoro. ... Una volta il film terminato, gli concedemmo il nulla osta" (67).

5 La discriminazione della donna era ben evidente anche sul piano legislativo: il codice penale italiano prevedeva per l'omicidio della moglie adultera l'attenuante della causa d'onore.

Questa era considerata un'attenuante specifica dell'omicidio, come veniva contemplato dall'articolo 587, fino alla sua abrogazione, avvenuta solo nell'agosto del 1981. Tale articolo era stato introdotto nel 1930, in pieno fascismo, dal codice Rocco, che modificava l'art. 337 del precedente codice Zanardelli. Il codice Rocco introduceva il delitto d'onore come reato autonomo, mentre il precedente costituiva solo un'attenuante generica. Ladulterio e il concubinato costituivano un reato penale in base agli articoli 559 e 560 del codice penale fino alla loro abrogazione nel 1968. Dai due articoli risulta che il tradimento è un reato penale solo per la moglie, mentre il coniuge è passibile di pena solo nel caso di convivenza more uxorio.

6 Cfr. Cecilia Dau Novelli 53-101.

7 Da un lato abbiamo la produzione dell'Istituto Luce con i suoi cinegiornali e documentari che offrono un'iconologia della donna e dei bambini stereotipata in linea con la propaganda di regime. 
guenza anche i bambini, come rileva, nel 1937, un editoriale di Cinema ${ }^{8}$. Tuttavia questi compaiono in alcuni melodrammi nel periodo dell'autarchia. Pur costituendo, come osserva Gian Piero Brunetta, riferendosi alla produzione filmica dei primi quarant'anni del secolo, "un ingrediente drammatico e narrativo di largo uso"

... sono visti come oggetti del paesaggio, figurine ritagliate dall'oleografia, elementi casuali e di disturbo rispetto all'intreccio. Sono afasici o bamboleggianti, alieni, mostri, scimmiette, piccoli animali ammaestrati da salotto che i grandi vorrebbero silenziosi come soprammobili, o caricature. Il bimbo è un optional narrativo, un condimento drammaticosentimentale da distribuire a piacere. ("Voci, sguardi, silenzi" 64)

Potremmo ricordare a volo d'uccello alcuni bambini e bambine, brevi apparizioni nella volta stellare del Ventennio: Ninni ne La canzone dell'amore (1930) di Gennaro Righelli, Annina ne La maestrina (1933) di Guido Brignone e remake di Giorgio Bianchi (1943), Maria in T'amerò sempre (1933 e remake del 1943) di Mario Camerini, Piero in É caduta una donna (1941) di Alfredo Guarini; oppure, in un ruolo più incisivo, Marina (Mariù Pascoli) ne La fuggitiva (1941) di Piero Ballerini, oppure Tonino ne La peccatrice (1940) di Amleto Palermi, Ombretta (Mariù Pascoli) in Piccolo mondo antico di Mario Soldati, "Totò" (Anna Maria Millo) in Zazà (1942) di Renato Castellani, Ada (Maria Sandri) ne La morte civile (1942) di Ferdinando M. Poggioli, Stefania (Giuseppina Fiore) in Nessuno torna indietro (1943) di Alessandro Blasetti9.

All'interno di una tematica più in sintonia con I bambini ci guardano, ovvero quella dell'adulterio femminile, troviamo una coppia di film della prima metà degli anni Trenta: Piccola mia (1933) di Eugenio De Liguoro (1895-1952) e Il canale degli angeli (1934) di Francesco Pasinetti (19111949). Il primo è un lungometraggio poco conosciuto del figlio di un regista abbastanza celebre dell'epoca del muto, Giuseppe De Liguoro ${ }^{10}$. Il secon-

8 Il fanciullo appare molto di rado: quando appare è privo della naturalezza vitale che interessa ed appassiona anime sane e cuori sani .... Fra i vari motivi che possono avervi concorso il cinema ha la sua grande parte di responsabilità nella diffusione di questo senso della vita che induce l'uomo a fuggire i pericoli di crearsi un focolare e che consiglia la donna, per "vivere la sua vita» secondo una espressione tanto comune quanto sciocca, ad evitare gli impedimenta della maternità (Cinema 165).

9 Raramente nei titoli di testa del film compaiono i nomi dei bambini a dimostrare la marginalità delle loro presenza.

10 Eugenio De Liguoro è un regista pressoché sconosciuto a cui non è stato riser- 
do invece è il film d'esordio dell'autore della prima Storia del cinema dalle origini ad oggi (1939), pubblicata in Italia ${ }^{11}$. Al centro delle trame due mogli e madri che sentono irresistibile l'attrazione per un eros che non coincide con agape, in quanto si prefigura come passione dei sensi, in netto contrasto con quello coniugale, identificato con figure maschili incapaci di comprendere le inquietudini e i desideri delle giovani mogli. Adriana, la bimba (Alessandra De Stefani) di Piccola mia, film di chiara imitazione americana, bionda e tutta boccoli, dal caratterino impertinente, ha un ruolo molto marginale, ma è lei a determinare la scelta drastica del padre (Ernesto Sabbatini), una volta scoperto l'adulterio della moglie Maria (Germana Paolieri).

Le parole rivolte dall'uomo alla moglie sono inequivocabili: "Immagino che tu avrai ben calcolato tutte le conseguenze...ora che tu hai scelto un'altra via, devi andare per quella strada. Di me non parlo, perché a me tu hai rinunciato già da un pezzo, ma si tratta ora di rinunciare anche a tua figlia ... desidero che mia figlia cresca accanto a persone per bene. Credevo che tu avessi pensato a questo e che il tuo nuovo amore ti ripagasse di tutto...”. E più avanti: "[Maria] ha dimenticato troppo facilmente i suoi doveri. È stata una cattiva moglie e soprattutto una cattiva madre .... Io ho le mie idee e sono convinto che quando una madre dimentica fino a questo punto di essere madre, non ha più il diritto di ricordarsene."

Due le accuse imputabili alla moglie infedele: il comportamento irresponsabile, non meditato nelle sue conseguenze; la "sindrome della cattiva madre," quasi una malattia infettiva da cui tenersi lontani. Per ristabilire l'ordine che la morale corrente impone, è necessario che Maria operi una sorta di "rito di purificazione" attraverso il sacrificio della propria dimensione passionale sull'altare dell'affetto materno. È questa, alla fine, la scelta della protagonista, che rinuncia all'amore adultero - coinvolgente, anche se insicuro - per l'amore di madre. La bambina, nelle poche battute assegnatele, attende con sofferenza il ritorno di Maria, di cui non conosce le cause della partenza improvvisa. Il ruolo di Adriana è quello di accentuare le dolorose conseguenze prodotte nei figli dalla condotta "immorale" materna.

vato uno spazio nelle storie del cinema, ma solo poche righe: “...dopo una sporadica attività in Italia, si trasferisce in Cile dove lavora sia come attore che come regista" (Martinelli 465-466).

11 Pasinetti fu un critico e teorico, oltre che sceneggiatore e regista. Tra i suoi numerosi incarichi ricordiamo la fondazione e direzione de Il Ventuno, la rivista dei GUF, l'attività di docenza e direzione del Centro Sperimentale di Cinematografia. Fu inoltre il primo studente italiano a laurearsi in Storia del cinema all'Università di Padova. 
La bimba infatti chiede con insistenza al padre se la madre le abbia scritto esprimendo il suo dolore di bambina privata di un affetto forte e insostituibile: "Papà è arrivata la posta?/C’è la lettera della mamma?/Dice che viene presto?/Dice sempre che viene presto e poi non viene mai/ Hai scritto che le voglio tanto bene?/Hai scritto che le mando tanti bacetti? "

E qui la trama del film propone un altro topos, ad aumentare la tensione drammatica: la malattia della piccola. Quasi una "somatizzazione da abbandono" che colpisce la figlioletta, dopo aver lungamente atteso il ritorno della madre ${ }^{12}$. La corsa forsennata di Maria, in auto, per recuperare il vaccino, noncurante del rischio di finire travolta da un treno, risolve con un gesto di abnegazione suprema il percorso di espiazione della protagonista.

La messa a repentaglio della propria vita, per la creatura cui ha dato la vita, serve a riconciliare la donna adultera con la parte lesa negli affetti (figlia e marito) e con la morale comune.

Piuttosto prevedibile l'esito della scena finale in cui Maria, ottenuto un tacito - e nel fuori campo - perdono del marito, abbraccia con trasporto la figlia con un enfatico "Piccola mia!"

Prende avvio sempre da una moglie sfiorata da inquietudini amorose, che mettono a repentaglio la solidità del ménage matrimoniale e producono sofferenza nei figli, anche il film Il canale degli angeli di Francesco Pasinetti. Tra opera di finzione e documentario ${ }^{13}$, il lungometraggio ambienta la vicenda in un contesto di forte realismo, in cui ognuno dei protagonisti è ben conscio del ruolo che la vita reale e le convenzioni sociali ha loro assegnato. Anna (Anna Ariani) - moglie e madre premurosa - nonostante l'attrazione per il simpatico marinaio, è restia a cedere al richiamo del cuore e dei sensi; il "Capitano" (Maurizio D'Ancora) - giovane libero e piacente - dopo le prime infruttuose avances, riconosce i limiti imposti dalla posizione sociale di Anna, nondimeno cerca di forzarli in nome di un sentimento che lo tiene legato a lei al punto di rinunciare al sogno di viaggi lontani per mare; Daniele (Ugo Gracci) — marito e padre affettuoso — assicura alla famiglia, con un lavoro di alta competenza, agiatezza economica e riconoscimento sociale, nel convincimento di aver così soddisfatto le esi-

12 Con forme narrative leggermente diverse tale topos si ripete anche ne Il canale degli angeli e ne I bambini ci guardano.

13 Quest'anima bifronte de Il canale degli angeli è stata messa in rilievo da Fasan: "Nella prima parte l'autore si concentra a delineare l'atmosfera e a presentare i personaggi assolutamente reali e realistici e colti nelle loro attività quotidiane, mentre nella seconda si sofferma a descrivere emozioni e conflitti interiori. Anche lo stile cambia in funzione di questa evoluzione: da documentaristico e di "contesto" diviene psicologico e intimistico." (182) 
genze della giovane moglie (e, forse, di averla in questo modo risarcita della considerevole differenza di età); Bruno (Pino Locchi), figlio attaccato alla madre e sensibile, dà libero sfogo alle manifestazioni emotive di chi vive un trauma affettivo. Rispetto alla pellicola di De Liguoro, in cui la bambina è funzionale a far ravvedere la madre e a ricondurla al suo ruolo di mater familias, qui è il bambino - con una parte da protagonista — a segnalare, come una cartina di tornasole, i vari passaggi della vicenda; è lui, con la sua emotività infantile, a dare concreta evidenza del dramma in atto, come ha sottolineato Gian Piero Brunetta:

Con dieci anni di anticipo rispetto a I bambini ci guardano di De Sica, si rende testimone e partecipe dell'azione un bambino, che prima rifiuta e poi capisce il dramma della madre e col suo silenzio le dimostra la sua complicità e la sua comprensione, e ottiene, grazie alla partenza dell'amante, la ricomposizione del nucleo familiare.... In più, come valore aggiunto che ancor oggi mi sembra regalare al film una freschezza intatta, c'è la sua capacità di porsi ad altezza di bambino, di misurare nei silenzi, sguardi, e movimenti di fuga di Bruno la sua angoscia per ciò di cui è testimone, il suo dramma che viene interiorizzato e somatizzato, ma che lo porta anche alla consapevolezza che il pericolo della distruzione del nucleo familiare è scongiurato dalla partenza del marinaio. ("Magia veneziana" 312-13)

È Bruno il vero antagonista del “Capitano.” È Bruno a cogliere i turbamenti emotivi della madre (il padre non è in grado di percepirli perché concentrato sul lavoro, o fiducioso della moglie, o - più semplicemente incapace). È Bruno a porre in atto tutte quelle "strategie di seduzione" che un figlio adotta per attirare su di sè l'attenzione perduta: la malattia (subito dopo l'episodio del bacio rubato), la rivalità (interrompe bruscamente i rapporti fino ad allora amichevoli con il "Capitano"), la complicità (mantiene il segreto del bacio e non ne informa il padre, che pure coglie il disagio del figlio: "Mi sembra che Bruno sia diventato più serio, troppo serio per la sua età...ho l'impressione che abbia qualcosa da nascondere"), la vendetta (rifiuta quasi infastidito le attenzioni di Anna, soprattutto ogniqualvolta la scopre in compagnia del marinaio); ricerca invece l'affetto del padre, come compensazione sì a "un'affettività negata" ("Magia veneziana" 178), ma forse anche come forma di punizione affettiva nei confronti della madre, la lusinga (ritorna affettuoso con Anna alla notizia che il "Capitano" ha deciso all'improvviso di imbarcarsi, quasi a compensare con il suo amore sincero, l'affetto destinato a finire con la partenza del rivale). Ma soprattutto Bruno — come dieci anni più tardi Pricò — tiene tutto il dolore dentro di sé ed è incapace di esternare con parole il suo turbamento di fronte alla possibile 
perdita della madre per un altro uomo. La massima tensione emotiva è proprio nel finale, quando la madre esce di casa e lo lascia solo ad attendere il padre per il pranzo: "Ho già preparato tutto. Non state ad aspettarmi. Io vado un momento fuori." Il "Capitano" l'aspetta fuori dalla casa e il bambino ha compreso che il pericolo di perdere la madre non è del tutto scongiurato. La donna potrebbe andarsene e non tornare più: anteporre l'amore per il giovane marinaio a quello per il figlio.

Anna, come Maria in Piccola mia, infatti è dibattuta tra queste due diverse tensioni amorose: per il figlio e per il giovane innamorato. Non sappiamo se nella scelta finale sia più determinante l'affetto per Bruno o la delusione per una promessa d'amore tradita. Il clima di incertezza è palpabile: il marito, di ritorno a casa, non si capacita dell'assenza, e nel suo inquieto conversare col figlio la nomina di continuo, quasi a evocarne la presenza; Bruno, benché affamato, non tocca cibo e attende con ansia sempre crescente. Non fa menzione del "Capitano," non risponde alle domande del padre preoccupato per il ritardo della moglie ("Ma dov'è andata la mamma?/ Ma non ti ha detto dove andava?"). Bruno non è meno preoccupato del padre, che di fatto non sa nulla. Solo che preferisce tenere il segreto per sé e distrarre il padre con inutili tentativi di stemperare la tensione dell'attesa: "Sai papà, oggi mi hanno promosso caposquadra e domenica mi daranno i gradi/ Papà la nave!/La nave grande che va in America”

La comparsa di Anna sull'uscio di casa, salutata dall'abbraccio festante del figlioletto ("Mamma, mamma mia! "), ristabilisce di colpo il clima di tranquilla consuetudine del pasto serale. L'affetto di madre è alfine prevalso sulle pulsioni amorose, il dovere del cuore sulla voluttà dei sensi. Insomma nei due film il deragliamento emotivo è vinto dalla forza dell'amore materno che riesce ad avere il sopravvento sulle pulsioni ed esigenze affettive e a salvare l'unione familiare. È comunque Bruno nella pellicola di Pasinetti a segnalare un interesse maggiore degli autori per il mondo dell'infanzia, pressoché marginale nelle pellicole di regime.

\section{I bambini ci guardano: Vittorio De Sica e l'incontro con " $\mathrm{Za}$ "}

I bambini ci guardano fa da capofila ad una serie di opere con bambini protagonisti dell'azione filmica ${ }^{14}$. Il merito di ciò si deve a Cesare Zavattini,

14 Pricò, il protagonista del film, è uno dei tanti ritratti infantili usciti dalla penna prodigiosa dello sceneggiatore emiliano. Sarà infatti proprio "Za" a scrivere il copione di Sciuscià (1946) e di Ladri di biciclette (1948), capolavori della grande stagione neorealista. Mentre ne I bambini ci guardano la Storia rimane sullo sfondo ed è percepita soltanto dall'impossibile unità della famiglia, alla base, 
padre teorico del neorealismo, che trova in De Sica un compagno di strada in perfetta sintonia con il suo modo di osservare il mondo degli adulti attraverso uno "sguardo- bambino." Il film è un adattamento del romanzo di Cesare Giulio Viola Pricò, un racconto breve ma intenso, il cui contenuto colpì in modo particolare il regista da indurlo a trasporlo sullo schermo $^{15}$. Questa la sua testimonianza

E il romanzo di Cesare Giulio Viola mi piacque per questo bambino che viveva in un ambiente, in una società ipocrita, bugiarda. La piccola borghesia che io descrivevo mi interessava per la sua meschinità e per la sua miseria morale. Ecco, il mio bersaglio era un fatto più importante (un fatto morale, o sociale), più importante che le vecchie commedie. (Savio 489)

"Za" e De Sica si erano conosciuti a Verona nel 1935 quando il regista recitava in Darò un milione, diretto da Mario Camerini. In seguito i due si incontrarono a Milano nel 1939 e Zavattini propose al regista, che lo acquistò, un soggetto dal titolo Diamo a tutti un cavallo a dondolo, che non divenne mai un film. Lo sceneggiatore emiliano venne poi interpellato da De Sica per mettere mano, in forma anonima, al copione di Teresa Venerdi (1941), un film appartenente alla produzione di regime con l'happy ending scontato. Il reale sodalizio tra i due però si concretizzò con I bambini ci

secondo l'etica fascista, della compattezza della Nazione, in Sciuscià i lustrascarpe Pasquale e Giuseppe e Bruno in Ladri di biciclette sono privati della loro fanciullezza dal mondo degli adulti che li costringono a lavorare per mantenere la famiglia. In tutti i tre film possiamo perciò constatare lo statuto debole della famiglia ed in particolare della figura paterna ne I bambini ci guardano e in Ladri di biciclette. Come ha scritto giustamente Giaime Alonge: "E proprio nella natura malferma del potere paterno risiede la profonda differenza tra le opere di De Sica e molti film di quel cinema dei bambini, che - da Zero in condotta (Zèro de conduite, 1933) di Vigo a Gli anni in tasca (L'argent de poche, 1976) di Truffaut — attraversa la storia della settima arte. ... In De Sica, invece, i bambini sono costretti a una precoce assunzione di responsabilità, perché non esiste una figura paterna che si prenda cura di loro. Nei film di De Sica, i figli devono presto farsi uomini, perché i loro padri - quando sono presenti - sono regrediti a una condizione infantile." (Alonge 79).

Sarà anche Roberto Rossellini con Roma città aperta (1945) ad assegnare all'infanzia un ruolo attivo, da protagonista. Marcello, il figlio della sora Pina, è già un piccolo uomo in grado di comprendere il significato della tragedia collettiva e privata che lo ha colpito.

15 De Sica scrisse la sceneggiatura assieme a Gherardo Gherardi, Margherita Maglione, Adolfo Franci, Cesare Giulio Viola, l'autore del romanzo. 
guardano in cui Zavattini ammise di aver realizzato per la prima volta un personaggio "vivo" e non un manichino

Il racconto si chiamava Pricò, e ne traemmo I bambini ci guardano. Era forse la cosa più bella che avesse scritto Giulio Cesare Viola, che in altre sedi non era il mio autore. Di quel racconto ero però disposto ad apprezzare la qualità umana e poi cinematografica - un racconto semplice, diretto, elementare, un racconto di valore. (57)

Fu proprio Zavattini a scegliere un titolo diverso da quello del testo letterario, traendolo da una rubrica giornalistica, dal titolo I nostri bambini ci guardano, da lui diretta sulla rivista Grazia e rivolta perciò ad un pubblico femminile. Il lavoro di riscrittura libera la pagina scritta da quell'atmosfera sensualmente voluttuosa di derivazione dannunziana e crepuscolare ${ }^{16}$. La alleggerisce degli eccessi patetici, procedendo con un lavoro di sottrazione e raffreddamento delle scene più enfatiche e melodrammatiche, per ricavare un copione in cui i sentimenti dei personaggi vengono espressi più che dalle parole - dallo scambio di sguardi, dalla gestualità trattenuta e dai silenzi carichi di pudore.

\section{Un melodramma della modernità}

I bambini ci guardano uscì sul grande schermo accompagnato da feroci polemiche e non rimase molto nelle sale. La critica dell'epoca lo considerò un'opera che tendeva alla puntualità veristica, alla precisione del documentario: "figure e costumi della moderna borghesia impiegatizia in un intreccio umilmente solito." (Palmieri 797). Con il passare del tempo, gli studiosi iniziarono a rivalutare questo lavoro e a considerarlo, assieme a Quattro passi tra le nuvole (1942) di Alessandro Blasetti e a Ossessione (1943) di Luchino Visconti, un'opera che anticipava la stagione del neorealismo. In realtà questo film, assieme a qualche altro del Ventennio fascista, stabilisce un distacco dalla produzione coeva per il coraggio dimostrato dal regista di affrontare tematiche considerate tabù e pertanto escluse dal grande schermo. In particolare i tre lungometraggi menzionati hanno in comune la crisi dell'istituzione familiare. In anni di repentini

16 In proposito vale la pena citare quanto ha scritto De Santi: "Si faccia tanto di pensare alla dilabente piagnucoleria del bambino, che rivela di certo una ferita, un trauma aperto. Poi si metta al suo posto il piccolo fanciullo querulo e sentimentale di Corazzini, aduso a rovesciarsi le lacrime addosso e a compatirsi. Si vedrà allora come il soggetto principale del libro, il bimbo appunto, possa essere agevolmente accluso a quel clima sentimentale." (31) 
mutamenti storici, anche la produzione cinematografica fascista sembra vada perdendo quel monolitismo che l'aveva fin qui caratterizzata.

La vicenda raccontata ne I bambini ci guardano è un concentrato di tematiche osteggiate vivacemente dalla propaganda fascista: adulterio della moglie-madre, suicidio dell'innocente, abbandono della prole indifesa, infanzia infelice ${ }^{17}$. Tutto trattato però col consueto garbo - a volte leggero, a volte drammatico - che riconosciamo a De Sica e che alleggerisce di molto la durezza dei temi considerati.

In realtà è lo stesso taglio narrativo a renderli meno incombenti, perché l'attenzione del regista è concentrata su altro: le aberrazioni e i guasti che può generare la collettività umana quando confonde morale e moralismo, rigore e intolleranza. Allora diventa un'umanità impicciona, malevola e maldicente, che interagisce e agisce in modo inesorabile e brutale sulle psicologie più fragili. Diventa il motore di comportamenti e scelte molto spesso dagli esiti drammatici.

È il caso di Andrea (Emilio Cigoli) che si vede costretto, a ogni allontanamento della moglie Nina (Isa Pola), a tenere lontano da casa il figlio Pricò $^{18}$ (Luciano De Ambrosis) per evitargli gli immancabili pettegolezzi; e in questa ristrettezza mentale dell'uomo si misura il divario con Roberto (Adriano Rimoldi), il rivale, del tutto incurante, come del resto Nina, dei giudizi della gente. Andrea rivela le debolezze del piccolo borghese celebrato dalla propaganza fascista: marito e padre di famiglia esemplare negli intenti, $\mathrm{ma}$ in realtà incapace di capire le reali esigenze della donna amata. Le decisioni dell'uomo sono sempre determinate dal timore del giudizio altrui e mai da un'esigenza interiore di scandagliare le ragioni delle fughe della moglie.

Quando l'azione del film ha inizio, il legame extra coniugale (con allontanamenti e riconciliazioni frequenti) tra Nina e Roberto è già in corso da due anni, segno che non è l'adulterio l'argomento clou della vicenda, ma le scelte affettive che questo comporta. Quasi fossimo di fronte al sequel delle trame esaminate in precedenza (Piccola mia e Il canale degli angeli), I bambini ci guardano prende avvio lì dove queste si sono fermate (la partenza-abbandono dell'amante). Il cinema italiano mai fino a quel momento ci aveva offerto immagini così eloquenti ed esplicite e allo stesso tempo misurate e pudiche di un adulterio, come ha scritto Gualtiero De Santi

17 Eloquente quanto scrive Gili a proposito dell'adulterio riferendosi al film di Vittorio De Sica I bambini ci guardano (1943): “... se l'adulterio esiste, è ammesso solo per l'uomo; l'adulterio della donna è l'apice dello scandalo poiché distrugge la famiglia e rimette in discussione la superiorità maschile" (59-60).

18 Pricò vuol dire precoce e quindi è un nomen omen, dato che questo bambino conosce molto presto la sofferenza. 
In questo senso il film ha la forza di dare veste metaforica alle crisi della società italiana conculcata dal fascismo, ma anche ad un trapasso determinante e essenziale verso un'altra condizione, che però, nei Bambini ci guardano (a differenza che nei film del neorealismo maturo), affonda in una disperazione non riscattata da una prospettiva di speranza. (38)

\section{La dialettica dello "sguardo-bambino" ne I bambini ci guardano}

I bambini ci guardano possiede numerosi punti di contatto con la vicenda raccontata da Pasinetti ne Il canale degli angeli: Pricò, come Bruno, infatti, attraverso la sua condotta esprime la tempesta emotiva che lo colpisce non appena comprende che le pulsioni amorose della madre verso un altro uomo rappresentano un pericolo non solo per la sua famiglia, ma soprattutto per lui, legato da un sentimento esclusivo e assoluto alla figura materna. A differenza del film-documentario del regista veneziano, De Sica rifiuta l'edulcorato lieto fine in nome di un'onesta e verosimile rappresentazione della famiglia alla fine degli anni Trenta. Al giro di boa del Ventennio, "la famiglia fascista non aveva più quasi nulla di quella tradizionale; forse solo il richiamo al numero dei figli e all'autorità paterna ne facevano un istituto esteriormente simile al passato. Era un soggetto politico che il regime tentava di utilizzare e di controllare in tutti i modi e che in realtà sfuggiva alla rete che gli era stata gettata addosso" (Dau Novelli 125).

I bambini ci guardano radiografa dunque senza pietismi proprio lo sfaldamento dell'unione familiare con le inevitabili ripercussioni che ciò comporta sulle esistenze dei protagonisti. In particolare sul piccolo Pricò, disarmato e infelice di fronte al fallimento del matrimonio dei suoi genitori.

Fin dall'inizio del film la macchina da presa sta addosso al bambino per scendere nei recessi della sua interiorità e captare nei suoi comportamenti le reazioni emotive agli eventi dolorosi che progressivamente si trova a dover vivere. Il primo è quando si trova al parco assieme alla madre e sta correndo spensierato con il suo monopattino, "padrone del suo spazio e di un tempo scandito da abitudini rassicuranti” (Caprioglio 43). Vede all'improvviso, in campo lungo, la madre assieme a Roberto, che quasi sicuramente conosce. Preso da una forte gelosia si avvicina alla coppia e attira le attenzioni della madre per andare via, rifiutandosi di stringere la mano dell'uomo. "Pricò ama come tutti i bambini essere padrone, e per fare questo, esercita un controllo costante su tutte le cose che gli appartengono. Non gli sfugge quindi il cambiamento del viso materno, quella persona estranea che entra e può minacciare il suo universo perfetto."(43)

Il pianto della madre e la menzogna alla sua domanda ("Mamma piangi?”) trasforma il suo sorriso in tristezza. 
Il dramma familiare è già tutto raccontato in quello "sguardo-bambino" che osserva gli eventi con una capacità di penetrazione sempre più acuta e consapevole. Nel momento in cui Nina abbandona - sembra non per la prima volta - il tetto coniugale, il bambino "punta gli occhi, febbrilmente, ora sul padre ora su Agnese, in cerca di un gesto chiarificatore" (Prudenzi 202). Andrea si dimostra inadeguato a fronteggiare la drammaticità della situazione: non sa giustificare davanti al figlio l'assenza di Nina, che solo il buon senso della governante Agnese (Giovanna Cigoli) riesce in qualche modo a rendere meno dolorosa con una frase pronunciata con commozione e a denti stretti "Mamma non c'è. È partita." Subito si scatena il pettegolezzo dei condomini e l'aspra critica della sorella di Nina e della suocera nei riguardi del gesto della donna. L'unico a non emettere sentenze su Nina è Pricò che tuttavia sente la mancanza della madre più di tutti. Per uno scherzo della sorte il bimbo, sballottato tra zia materna e nonna paterna, assiste da osservatore passivo o soltanto curioso alle vicende amorose dell'universo muliebre con cui entra in contatto, un universo che tuttavia accentua, anziché alleggerire, il "vuoto sentimentale" (207), lasciato dalla partenza di Nina.

Non può mancare neppure qui il topos della malattia che mette a rischio la vita di Pricò. La reazione di Nina è quella di madre: accorre al capezzale, anche se sembra quasi accettare di rientrare nell'ambito familiare solo per le insistenze del piccolo a rimanere, insistenze che spingono il marito a riaccoglierla. Il ménage coniugale si fonda però su "una armonia apparente, tenuta insieme dalla volontà del bambino, che, in un disperato scambio di sguardi con il padre, si dichiara abbastanza forte per colmare le distanze che separano i genitori" (207). La riacquisita condizione di madre sembra aver posto fine alla storia d'amore tra Nina e Roberto: i tentativi di riavvicinamento del giovane - anche energici e ultimativi — vengono respinti in nome di una maternità sofferta ma consapevole; il rapporto con Andrea è lentamente recuperato in una serena convivenza, anche se guardinga e quasi impacciata. Uno dei momenti di possibile avvicinamento è rappresentato dalla festa della mamma, un evento che viene favorito dalla fantesca dal cuore d'oro, Agnese, che convince Andrea a dare dei soldi al bambino per comprare dei fiori per Nina, perché così si è sempre fatto. Andrea accetta per la gioia del bambino e cerca di rendere piacevole la festicciola in terrazza con tanto di fiori e di regalo per la moglie. Nonostante il clima rasserenato, non c'è mai un gesto di intimità o un contatto fisico tra i due coniugi e l'affetto è colto di riflesso attraverso il rapporto con il figlio.

Pricò, Nina e Andrea siedono su tre lati di una tavola imbandita, senza che differenti distanze li separino. Il piccolo risulta al centro dell'inqua- 
dratura, ribadendo, se ce ne fosse bisogno, che è lui il cuore dell'azione e che la sua vicinanza ai genitori è transitoria misura del ritrovato legame affettivo tra i due. Tutta l'azione è accompagnata da sguardi, da trasalimenti carichi di pudore, quasi che il manifestare affetto sia più difficile che dimostrare odio o disaffezione. (203)

Un'altra occasione di serenità per Pricò e i suoi genitori è rappresentata dalle vacanze ad Alassio, una parentesi felice che sembra preludere ad una riconciliazione definitiva tra i due coniugi. Ma sia Nina che Andrea sono incapaci di comunicare i propri sentimenti, di dichiarare le proprie fragilità affettive. E infatti la ricomparsa di Roberto ad Alassio, in un ultimo disperato tentativo di convincere Nina a fuggire per sempre con lui, dà un taglio netto al precario equilibrio riacquistato. $\grave{E}$ ancora una volta lo sguardo di Pricò a scoprire in campo lungo l'uomo mentre abbraccia la madre sulla spiaggia: "Contrariamente a quanto accaduto in precedenza, non vi è gioco di occhiate tra madre e figlio: si tratta del palesamento di un iniziale distacco, che prenderà sempre maggiormente corpo" (210).

La donna ormai ha scelto di lasciare marito e figlio per realizzarsi come donna; l'abbandono avviene nel fuori campo e la scoperta della verità è lasciata, ancora una volta, agli sguardi di Andrea e di Agnese che si accorgono che il bambino è tornato dalle vacanze soltanto con la sua valigia. La madre con un pretesto non è salita in casa e se n'è andata, incapace di esprimere il suo malessere interiore, la sua impossibilità di rinunciare all'uomo amato per Pricò. Spetta ancora ad uno scambio di sguardi tra padre e figlio, accompagnato da poche ed essenziali parole, lo svelamento della verità: Andrea chiede al figlio se lui e la madre siano rimasti sempre assieme, loro due soli, dal giorno in cui è partito per tornare a Roma. La risposta del bambino si fa attendere. Non riesce più a guardare negli occhi suo padre. Abbassa lo sguardo imbarazzato. L'amore per Nina è talmente forte da indurlo a mentire sia per non arrecare un dolore al padre sia per una forma di protezione e complicità con la madre, alimentata dall'illusione infantile che possa ritornare ancora da lui. Sempre più in difficoltà nel nascondere la verità, di fronte alle domande incalzanti del padre, si addossa la colpa della condotta della madre: “... mamma si è ammalata perché io sono stato cattivo ... sì perché io un giorno sono scappato ... sì perché volevo venire da te col treno." Solo questa spiegazione è sufficiente ad Andrea per avere la conferma che la moglie si è incontrata con l'amante: "È venuto forse qualcuno laggiù quando non c'ero? Chi è venuto? " Il dialogo tra padre e figlio, giocato su primi e primissimi piani, che mettono in risalto la tempesta emotiva di entrambi, culmina con l'ennesima bugia di Pricò: "Nessuno, papà." Andrea crede di far capitolare il figlio affermando che 
Nina gli ha riferito tutto in una lettera, ma non ottiene un'esplicita confessione dal figlio ("Tu sei fuggito perché è venuto qualcuno. Rispondi”), bensì uno sguardo sofferente, che si proietta più che verso il padre, dentro la propria interiorità oltremodo ferita. Pricò dunque si sente tradito

dalla madre che preferisce il suo amante e lo lascia solo e dal padre che gli tende un tranello servendosi di lui per sapere una verità che ha sempre avuto paura di verificare. In quell'ostinato silenzio c'è la disperazione di aver perso quella dignità che lo faceva sentire complice dell'uno e dell'altro. (Caprioglio 44)

Ma non è questo l'ultimo dolore per il bambino: Andrea non se la sente di crescere da solo il figlio e preferisce metterlo in collegio. Anche in queste ultime sequenze del film notiamo lo sguardo di Pricò che cerca di trattenere Andrea, che lo ha lasciato da solo all'interno del vasto e freddo istituto con persone sconosciute. La corsa e il grido disperato del bambino lungo l'ampio scalone si concludono con uno sguardo di indicibile dolore: Pricò chiama il padre per non essere abbandonato anche da lui. L'uomo però ha già deciso e continua a scendere le scale senza voltarsi, senza ricambiare lo sguardo del figlio, che lo osserva angosciato.

Nell'ultima sequenza del film il distacco dalla madre diventa definitivo: alla notizia della perdita del padre, Pricò è convocato dal rettore del convitto nel suo ufficio. Vestito nella divisa del collegio, si trova davanti la madre, vestita a lutto, e Agnese. Nonostante l'invito del prete e di Agnese di andare dalla mamma, il bambino in lacrime, in un primo momento, si getta nelle braccia dell'anziana cameriera, e poi di fronte alla figura materna rimane impietrito. La guarda in lacrime, mentre lei riesce solo a pronunciare commossa e imbarazzata "Pricò." La donna non gli chiede perdono, come nel romanzo, in cui si inginocchiava davanti al figlio. Rimane invece come in trance, in attesa che il figlio faccia la prima mossa. E questi sempre piangendo decide di andare via, di tornare da padre Michele che lo attende in fondo al salone. Abbassa gli occhi e si distacca per sempre dalla madre: una conclusione per nulla melodrammatica ${ }^{19}$, anzi ripresa con estremo distacco dalla macchina da presa che inquadra i personaggi di proposito in campo lungo: "Il suicidio del padre è ormai un peso insopportabile. Pricò avanza nel totale inquadrato da lontano, con le volte alte e solenni del salone che lo rendono ancora più minuscolo. Su lui pesa un fardello troppo grande per un bambino" (De Santi, 41). Non accettando di trovare consolazione tra le braccia

19 Era stato girato un secondo finale con Pricò che abbraccia la madre, ma venne scartato. 
della madre, dimostra di non voler più soffrire, di non voler essere più "tradito proprio dalle persone a lui più care" (Caprioglio 44).

\section{Conclusioni}

Come abbiamo visto, pur essendo una presenza molto evanescente all'interno della produzione cinematografica di finzione dell'Italia fascista, i bambini si ritagliano una parte da protagonisti in alcuni film emblematici come Il canale degli angeli (1934) di Pasinetti e I bambini ci guardano (1943) di De Sica. Tra le due opere ci sono molti punti in comune, come lo sguardo con cui Bruno e Pricò assistono impotenti al tradimento extraconiugale delle proprie madri. I due piccoli assumono un atteggiamento protettivo nei riguardi della figura materna a cui sono legati da un rapporto viscerale e vivono con estrema sofferenza la possibilità di perderla. Bruno tuttavia riesce a mettere in atto tutta una serie di strategie che condurranno la madre a rinunciare all'attrazione per il giovane "Capitano" in nome dell'amore materno molto più forte, come del resto accade anche alla madre protagonista del coevo Piccola mia di De Liguoro. O forse i tempi non sono ancora maturi - a metà anni Trenta - per mostrare la crisi dell'istituzione familiare già una realtà di fatto, che il fascismo tuttavia preferisce nascondere per motivazioni ideologiche. Sarà I bambini ci guardano di De Sica a rivelare senza ipocrisie lo sfaldamento del nucleo domestico raccontando, grazie all'apporto di Zavattini, più che una storia di adulterio gli effetti devastanti che esso provoca. In particolare grazie allo sguardo di Pricò, sorta di fratello maggiore di Bruno, De Sica registra, con una capacità di introspezione inedita, l'inadeguatezza dei genitori di rapportarsi non solo tra di loro ma soprattutto con il figlio. Andrea non è più il canonico pater familias celebrato dalla propaganda di regime. Si rivela debole, introverso e incapace di comprendere le esigenze di Nina, come di occuparsi del figlio, proprio perché schiacciato dai pettegolezzi di un contesto sociale impietoso. Nina, dibattuta tra amore materno e realizzazione affettiva, sceglie quest'ultima, incurante del falso perbenismo che invece spinge all'autodistruzione il marito. A Pricò però spetta la scelta definitiva di fronte alla perdita dei suoi punti di riferimento affettivi: si rifiuta di trovare consolazione tra le braccia della madre perché ha perduto ogni forma di fiducia nel mondo degli adulti, in balia di pulsioni emotive per lui incomprensibili ma che sono la causa della sua infelicità. 


\section{Opere citate}

Alonge, Giaime. Vittorio De Sica. Ladri di biciclette. Torino: Lindau, 1997.

Brunetta, Gian Piero. "Voci, sguardi, silenzi: il viaggio del bambino nel cinema italiano." In Barbara, Corsi e Diego, Leoni (a cura di). Metafore d'infanzia. Il bambino nel cinema italiano. Rovereto: Edizioni Osiride, 1993. 64-73.

"Magia veneziana nello sguardo di un bambino," Appendice 2. In Erika Fasan. Dal Veneto minore ai fasti imperiali: il documentario italiano tra realismo e propaganda (1925-1945). Tesi di dottorato discussa all'Università di Padova nell'anno accademico 2008-2009, supervisore prof. Gian Piero Brunetta. 311-313.

Caprioglio, Donatella. "I bambini come ci guardano." I bambini ci guardano di Vittorio De Sica. Testimonianze, interventi, sceneggiatura (a cura di Gualtiero De Santi e Manuel De Sica). Roma: Editoriale Pantheon, 1999. 43-45.

Cardone, Lucia. Il melodramma. Milano: Editrice Il Castoro, 2012.

Dau Novelli, Cecilia. Famiglia e modernizzazione in Italia tra le due guerre. Roma: Studium, 1994.

De Santi, Gualtiero. "La cognizione del dolore." I bambini ci guardano di Vittorio De Sica. Testimonianze, interventi, sceneggiatura (a cura di Gualtiero De Santi e Manuel De Sica). Roma: Editoriale Pantheon, 1999. 31-41.

Dogliani, Patrizia. Il fascismo degli italiani. Una storia sociale. Torino: UTET, 2008.

Fasan, Erika. Dal Veneto minore ai fasti imperiali: il documentario italiano tra realismo e propaganda (1925-1945). Tesi di dottorato discussa all'Università di Padova nell'anno accademico 2008-2009, supervisore prof. Gian Piero Brunetta.

Gili, Jean A. Stato fascista e cinematografia. Repressione e promozione. Roma: Bulzoni, 1981.

"La naissance d'un cinéaste." In Orio Caldiron (a cura di). "Vittorio De Sica." Bianco e Nero, 36. 9/12, settembre-dicembre 1975: 50-65.

Grignaffini, Giovanna. "Il volto e la divisa. Luoghi del femminile nel cinema italiano degli anni Trenta." Giovanna Grignaffini. La scena madre. Scritti sul cinema. Bologna: Bononia University Press, 2002. 239-255.

Martinelli, Vittorio. “Giuseppe De Liguoro.” Dizionario dei registi del cinema mondiale. Gian Piero Brunetta (a cura di). Vol. 1, Einaudi: Torino, 2006. 465-466.

Nicoletto, Meris. Percorsi tra tradizione e modernità all'interno dell'universo fermminile nel cinema di regime (1929-1943). Tesi di dottorato discussa all'Università di Padova nell'anno accademico 2012-13, supervisore prof. Gian Piero Brunetta. 233-304.

Palmieri, Eugenio Ferdinando. "I bambini ci guardano" L'illustrazione italiana. 71. 51, 17 dicembre 1944. 797.

Prudenzi, Angela. I bambini ci guardano, De Sica, autore, regista, attore. A cura di Lino Micciché Venezia: Marsilio, 1992. 197-218.

Savio, Francesco. "Intervista a Vittorio De Sica”. In Cinecittà anni Trenta. Parlano 116 protagonisti del secondo cinema italiano (1930-1943). Vol. 2 (DEG-MOR), Roma: Bulzoni, 1979. 483-492.

Viola, Cesare Giulio. Pricò. Milano: Mondadori, $1943^{3}$. 
Zavattini, Cesare, "Intervista”. In Franca Faldini e Goffredo Fofi (a cura di). L'avventurosa storia del cinema italiano raccontata dai suoi protagonisti 19351959. Milano: Feltrinelli, 1979, 56-57.

Anonimo, "Editoriale." Cinema. 2. 17, 10 marzo 1937, 165.

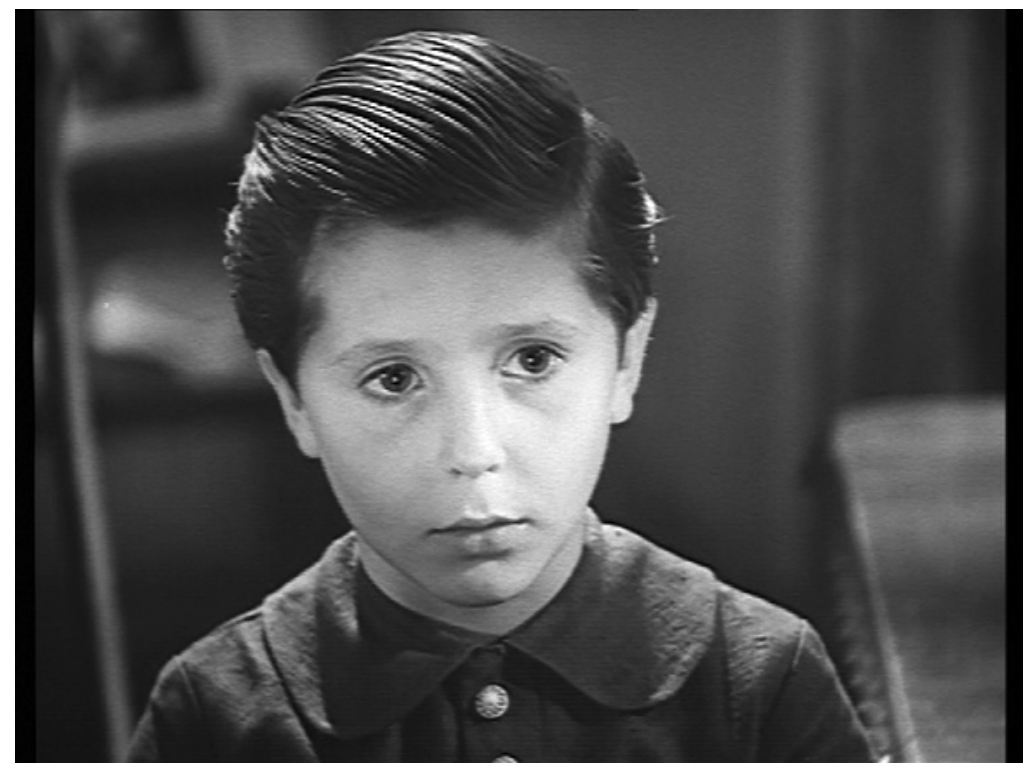

Foto 1. Pricò imbarazzato quando il padre lo interroga per sapere dove sia la mamma.

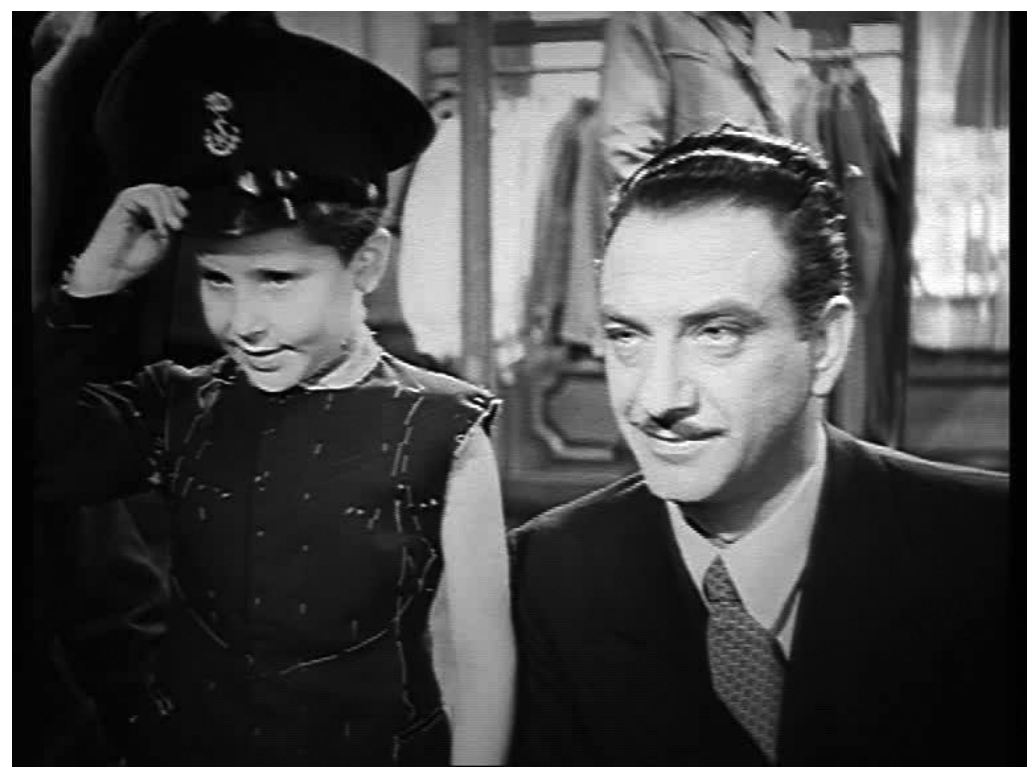

Foto 2. Il padre di Pricò fa confezionare l'uniforme per il bambino che va in collegio. 